\title{
Russian Space Program: Experiments in Solar-Terrestrial Physics
}

\author{
L.M.Zelenyi ${ }^{1}$, V.D.Kuznetsov ${ }^{2}$, Yu.D.Kotov ${ }^{3}$, A.A.Petrukovich ${ }^{1}$, \\ M.M.Mogilevsky ${ }^{1}$, K.A.Boyarchuk ${ }^{2}$, G.N.Zastenker ${ }^{1}$ and \\ Yu.I. Yermolaev ${ }^{1}$ \\ ${ }^{1}$ Space Research Institute (IKI), Profsoyuznaya 84/32, Moscow 117997, Russia \\ email: Lzelenyi@iki.rssi.ru \\ ${ }^{2}$ Institute of Terrestrial Magnetism, Ionosphere and Radio Wave Propagation (IZMIRAN), \\ Moscow Region, Russia \\ ${ }^{3}$ Moscow Engineering Physics Institute (MEPhI), Moscow, Russia
}

\begin{abstract}
We present a brief review of scientific milestones of the Russian Space Research Program for 2006-2015 in the field of solar and solar-terrestrial physics and describe several space projects: CORONAS-PHOTON, RESONANCE, CLIPPER, INTERHELIOPROBE, and THERION-F2.
\end{abstract}

\section{Introduction}

One of the most important scientific parts of the Russian Space Research Program is solar and solar-terrestrial physics. Objects of this research, Sun, solar wind, plasma environments of the Earth and other planets, are connected by hierarchy of the physical processes, transferring kinetic and electromagnetic energy from the Sun. Distinctive feature of such investigations is their integrated, multidisciplinary character, allowing to obtain substantially new knowledge on acting physical mechanisms and response of the near-Earth environment to the state of the interplanetary space. Therefore, this research is of the large practical interest for the space weather applications.

In this paper we present a brief review of several space projects which are planned to be included in Russian Federal Space Program for 2006-2015 and will join the international space science fleet (Zelenyi and Petrukovich, 2004):

- CORONAS-PHOTON, targeted at the studies of energy transformation and particle acceleration during solar flares;

- RESONANCE, targeted at wave-particle interaction studies in the Earth's magnetosphere;

- CLIPPER, devoted to monitoring of the solar wind and forecasting of the space weather;

- INTERHELIOPROBE, planned to observe solar atmosphere and internal heliosphere at the distance of 30 solar radii from the Sun;

- THERION-F2, targeted to determination of ionosphere and thermosphere properties on the basis of in situ and remote methods on the low-orbit satellite.

\section{CORONAS-PHOTON}

The main objectives of the project include following scientific problems:

- Physics of the Sun 


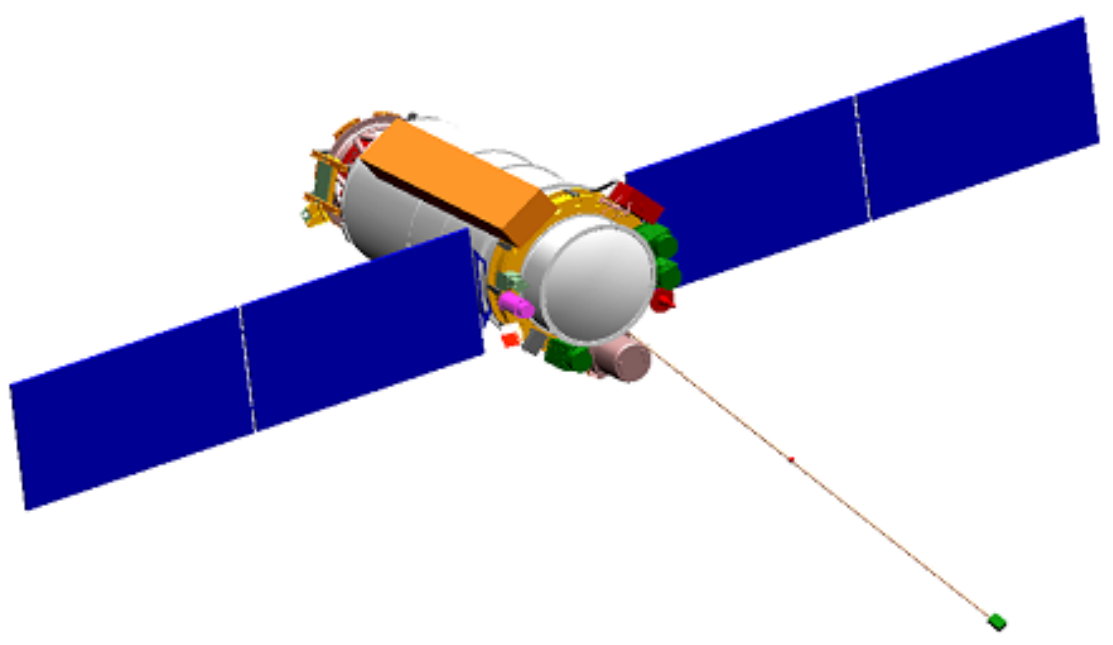

Figure 1. General view of the spacecraft CORONAS-PHOTON (METEOR 3M)

- Determination of distribution functions of accelerated electrons, protons and nuclei and their dynamics with a high time resolution;

- Research of difference in acceleration dynamics of electrons and protons (nuclei);

- Research of distribution function variations for high energy particles (up to a few $\mathrm{GeV})$;

- Research of interacting particle angular anisotropy by statistical analysis of radiation spectra and linear polarization parameters of hard X-rays;

- Study of directional effects in the region of high energy gamma radiation;

- Determination of mechanisms and requirements of electrons and protons acceleration in different flare phases, and parameters of propagation region of accelerated particles;

- Determination of elemental abundance in the region of gamma-ray production by gamma spectroscopy and capture of low energy neutrons in the solar atmosphere;

- Determination of radiation generation altitudes by observation of deuteron line weakening from limb flares;

- Determination of energy spectra view of accelerated protons and nuclei and dynamics of these spectra according to nuclear gamma-line ratio;

- Study of light elements generation (D, 3He, Li, Be) during flares;

- Solar-terrestrial physics

- Research of chemical and isotopic compositions of nuclei accelerated in flare on the Earth orbit, and also energy and temporal parameters of flare electrons and protons;

- Monitoring of the Earth upper atmosphere by absorption of extreme ultraviolet of the quiet Sun;

- Astrophysics

- Study of hard X-ray and gamma radiation from gamma-ray bursts;

- Study of X-ray radiation from the bright local sources along Ecliptic plane.

Moscow Engineering Physics Institute (MEPhI) is responsible for the scientific payload. Principle investigator is Dr. Yuri Kotov (MEPhI, kotov@mephi.ru). Spacecraft (see Fig. 1) and scientific payload weights are 1900 and $540 \mathrm{~kg}$. Orbit is circular, with hight 


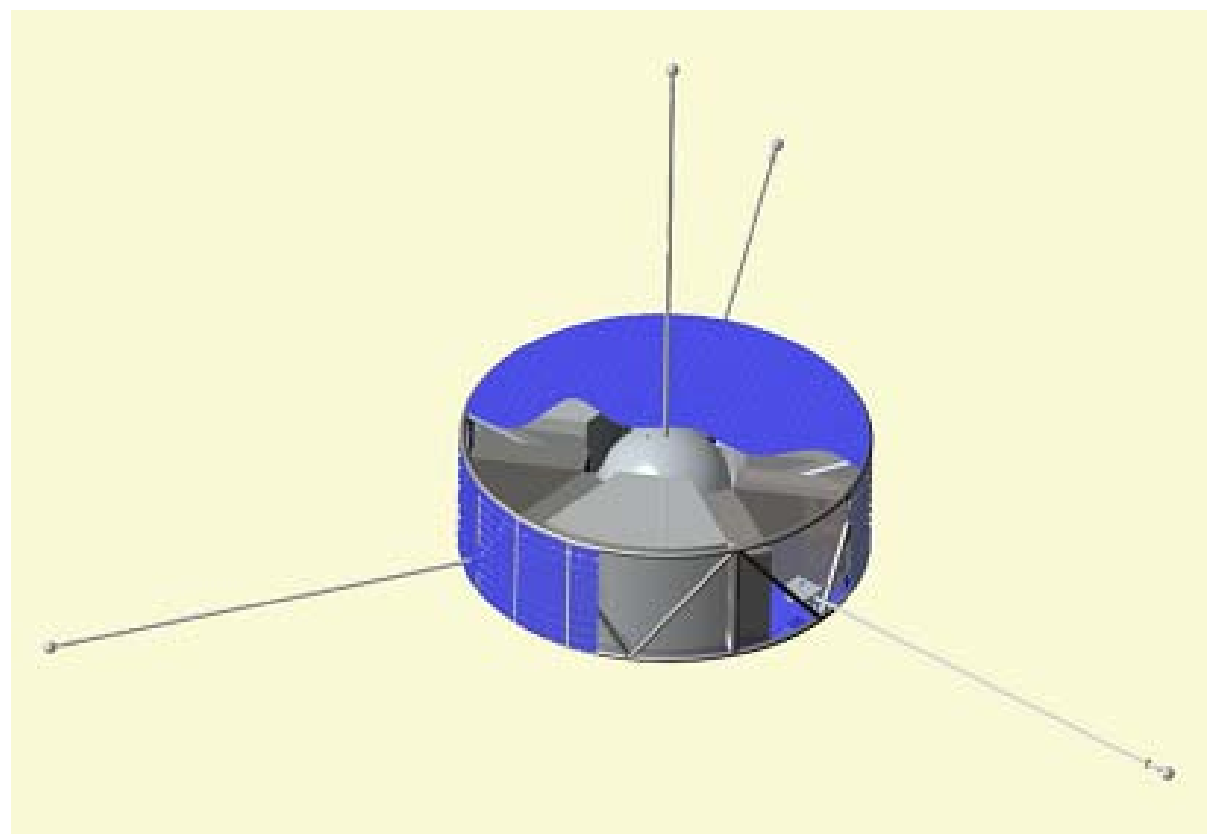

Figure 2. General view of the spacecraft RESONANCE

$500 \mathrm{~km}$ and inclination $82 \mathrm{deg}$. Nominal mission lifetime is 3 years (extended up to 5 years). Volume of scientific information stored per day is 8.2 Gbit. The mission WWW site is http://astro.mephi.ru/photon/photon.htm.

\section{RESONANCE}

Magnetospheric RESONANCE project (Mogilevsky, 2002; Demekhov et al. 2003) includes following main scientific goals:

- Long-term observations of the natural phenomena, localized in the selected flux tube:

- Dynamics of magnetospheres cyclotron maser,

- Ring current formation,

- Refilling of plasmosphere after magnetic storms,

- Study of the role of the small-scale phenomena in the global plasma dynamics.

- An artificial influence on the operation of magnetospheric maser:

- Artificial excitation and/or stimulation of wave modes;

- Modification of the flux of precipitating particles;

- Variation of maser Q-factor by the modification of reflection index in the ionospheric footprint of the selected magnetic flux tube.

Investigations in the frame of the second, "active", part, will focus on the joint experiments of the RESONANCE satellite(s) with ground-based HF heating facilities (HAARP and/or MURMANSK). We assume that parameters of the natural magnetospheric oscillatory system changes if powerful HF electromagnetic emissions heat the ionosphere and thus modulate the ionospheric mirrors. Phase and amplitude of magnetospheric oscillations, measured onboard the RESONANCE satellite, will be transmitted to the the heating facility, and used to modulate the HF radiation. In a case of in-phase modification, the amplitude of the natural oscillations should increase and inverse, antiphase, modification should decrease the oscillation. Such a unique experiment will help to 


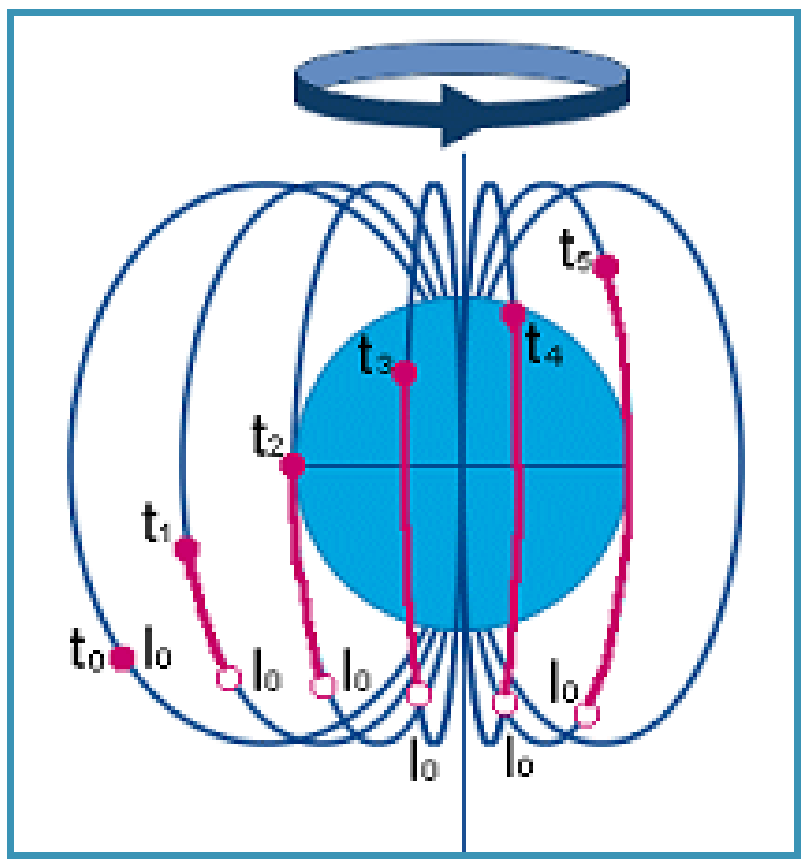

Figure 3. Trajectory of RESONANCE satellite

investigate important underlying principles in cyclotron maser theory and clarify the role of ionospheric mirrors in wave generation.

Spacecraft (see Fig. 2) and scientific payload weights are 550 and $100 \mathrm{~kg}$. The RESONANCE project has Magnetosynhronous orbit (apogee $28000 \mathrm{~km}$, perigee $500 \mathrm{~km}$, inclination 63.4 deg.) because the satellite moves along geomagnetic line during long time (Fig. 3) and spends more then 3 hours in the same flux tube $(L=5.5 \pm 0.15)$. Nominal mission lifetime is 5 years. The mission WWW site is http://www.iki.rssi.ru/resonance/.

\section{CLIPPER}

The main objectives of the CLIPPER project are

- independent continuous monitoring of the interplanetary environment in the interests of other solar-terrestrial experiments and space weather applications;

- investigation of spatial and temporal dynamics of the solar wind and interplanetary magnetic field in a wide range of their scales;

- development of real-time prediction techniques for the reliable short-term forecast of space weather.

Warnings of "dangerous" disturbances in the interplanetary space, performed on the basis of continuous observations in libration point L1 ( 1.5 million of $\mathrm{km}$ from the Earth), have almost $100 \%$-s' reliability, but their prediction time is $40-60$ minutes only. The spacecraft with total weight $30 \mathrm{~kg}$ and the solar sail of the order of $1000 \mathrm{sq} \mathrm{m}$ will be stopped by the solar light pressure near to the Sun-Earth line at about 3-4 million of $\mathrm{km}$ from the Earth (see Fig. 4), helping to increase prediction time up to 2-3 hours. Reliability of prediction will be better, if the CLIPPER project will include 2-4 spacecraft, placed in a tetrahedron formation with the distance between them of 100-200 thousand $\mathrm{km}$, in order to estimate spatial scales, uniformity and a configuration of plasma and magnetic disturbances. 


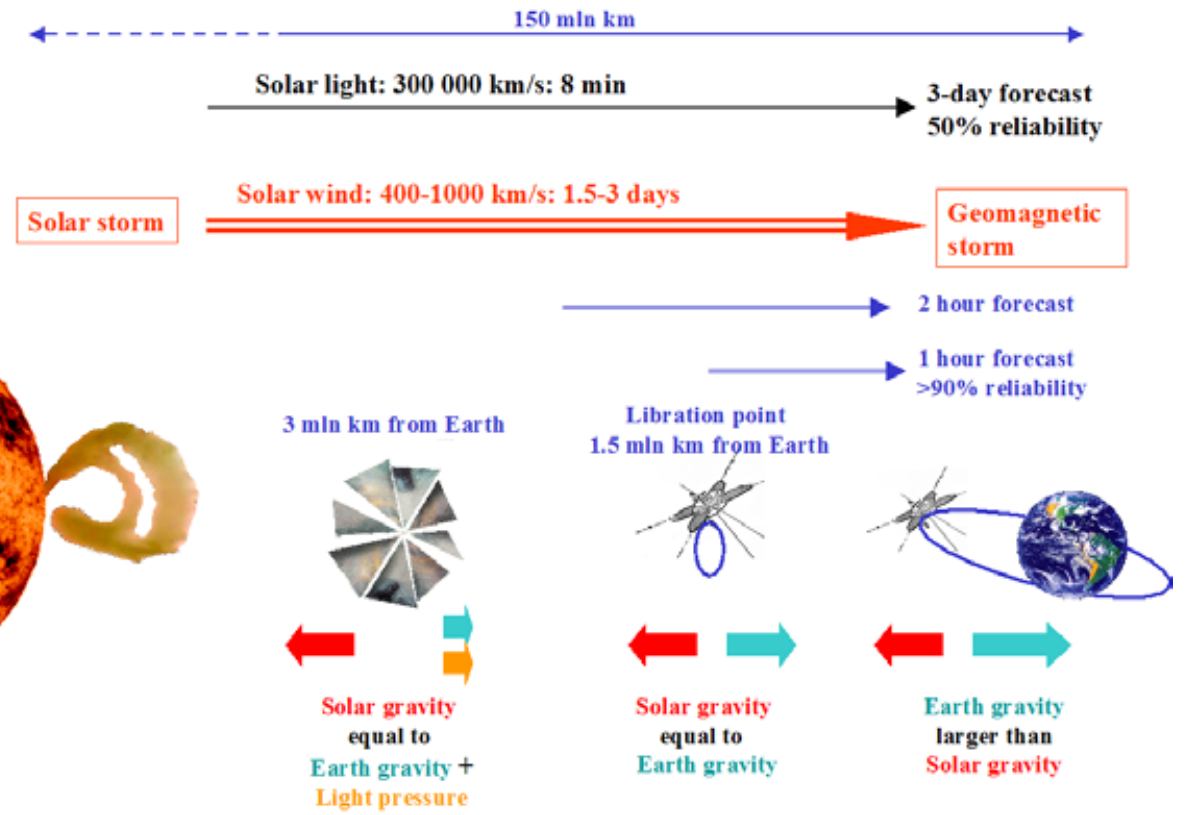

Figure 4. Schematic view of space monitoring by CLIPPER spacecraft

\section{INTERHELIOPROBE mission}

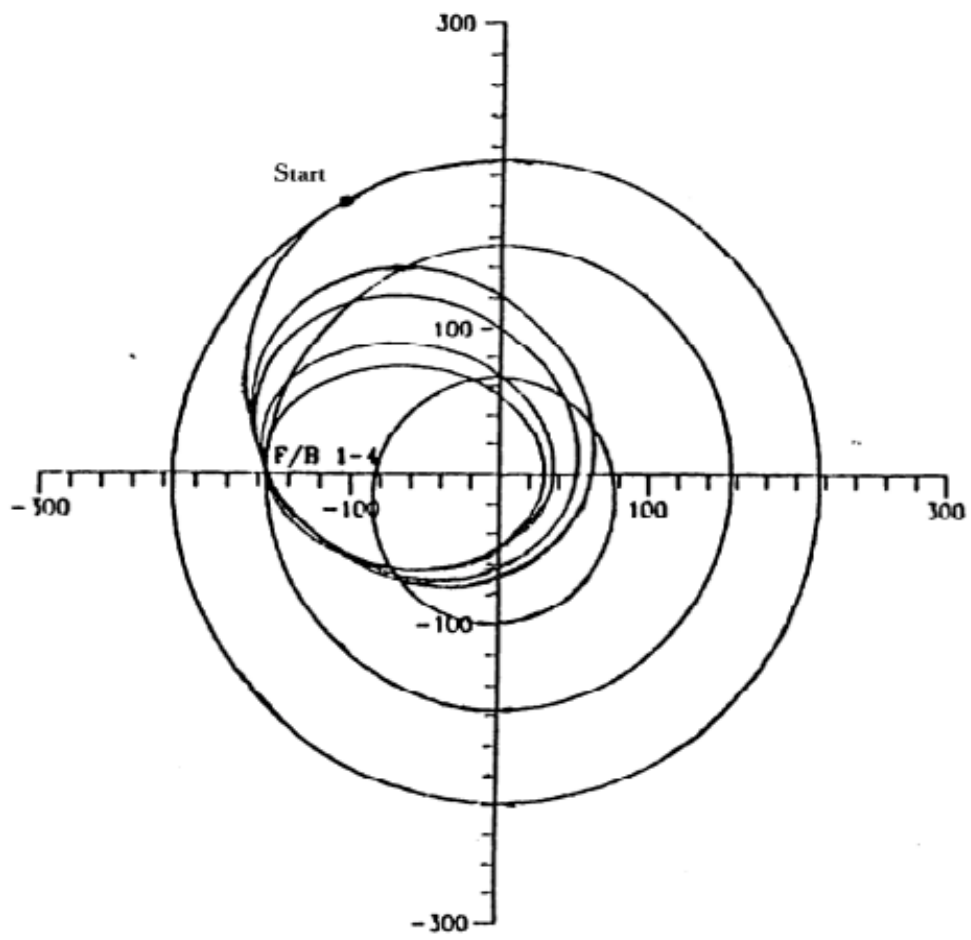

Figure 5. Trajectory of INTERHELIOPROBE spacecraft 
The INTERHELIOPROBE project (Oraevsky et al. 2001; 2002) includes direct interplanetary measurements and remote observations of the Sun and is aimed to sturdy following scientific problems of solar corona and heliosphere:

- To observe the solar atmosphere with a high time and spectral resolution for studying its fine structure and small-scale dynamics, including the potential outflow region of the variable slow solar wind, as well as for establishing the microplasma processes that determine the solar chromosphere and corona heating and the acceleration of the slow and fast solar wind,

- To study the active regions, flares, ejections, and other interplanetary disturbances, as well as of temporal and spatial correlations in the Sun and in interplanetary space during partial co-rotations of the spacecraft with the Sun.

- To determine the characteristics of the solar wind, plasmas, and magnetic field in the heliosphere regions, connected with the magnetoactive belt of streamers and coronal holes, that extend from the polar to the near-ecliptic latitudes,

- To study the plasma turbulence and the processes involved in acceleration, containment, and propagation of energetic particles in the solar environment,

- To obtain stereo images of solar features and to study their 3D space-time dynamics,

- To test and improve the remote and in-situ methods for forecasting the heliospheric weather and studying solar-terrestrial relationships.

Setting the spacecraft on the solar orbit with a perihelion of about $30 \mathrm{Rs}$ will require a number of gravity-assisted maneuvers (GM) near Venus. The trajectory consists of two consecutive parts: the perihelion lowering phase and the working orbit with a period of about 110 days. Fig. 5 illustrates one version of the mission, when a series of consecutive GM near Venus are realized at one and the same point. The use of a low-thrust engine will optimize the transition orbit and shorten to 13 months the time of reaching the working orbit at the first co-rotation point. The main ballistic scheme of the INTERHELIOPROBE mission uses the orbits in or close to the ecliptic plane. A slight inclination of the orbit towards the ecliptic plane is important for the study of the heliospheric current sheet and the IMF sector structure. A stronger inclination (up to $35 \mathrm{deg}$.), necessary for observation of the polar regions of the Sun and the high-speed solar wind, can be ensured by a GM near Venus and by using a low-thrust engine.

The general view of the INTERHELIOPROBE spacecraft construction with the deployed scientific payload is shown in Fig. 6. The preliminary mass report (full mass of 463 $\mathrm{kg}$ ) is as follows: the flight module - $320 \mathrm{~kg}$, the scientific payload - $60 \mathrm{~kg}$, the container for scientific instruments with the heat screens - $25 \mathrm{~kg}$, the adapter - $20 \mathrm{~kg}$, the mass reserve $38 \mathrm{~kg}$. The mission WWW site is http://www.izmiran.rssi.ru/projects/INTERHELIOS. 

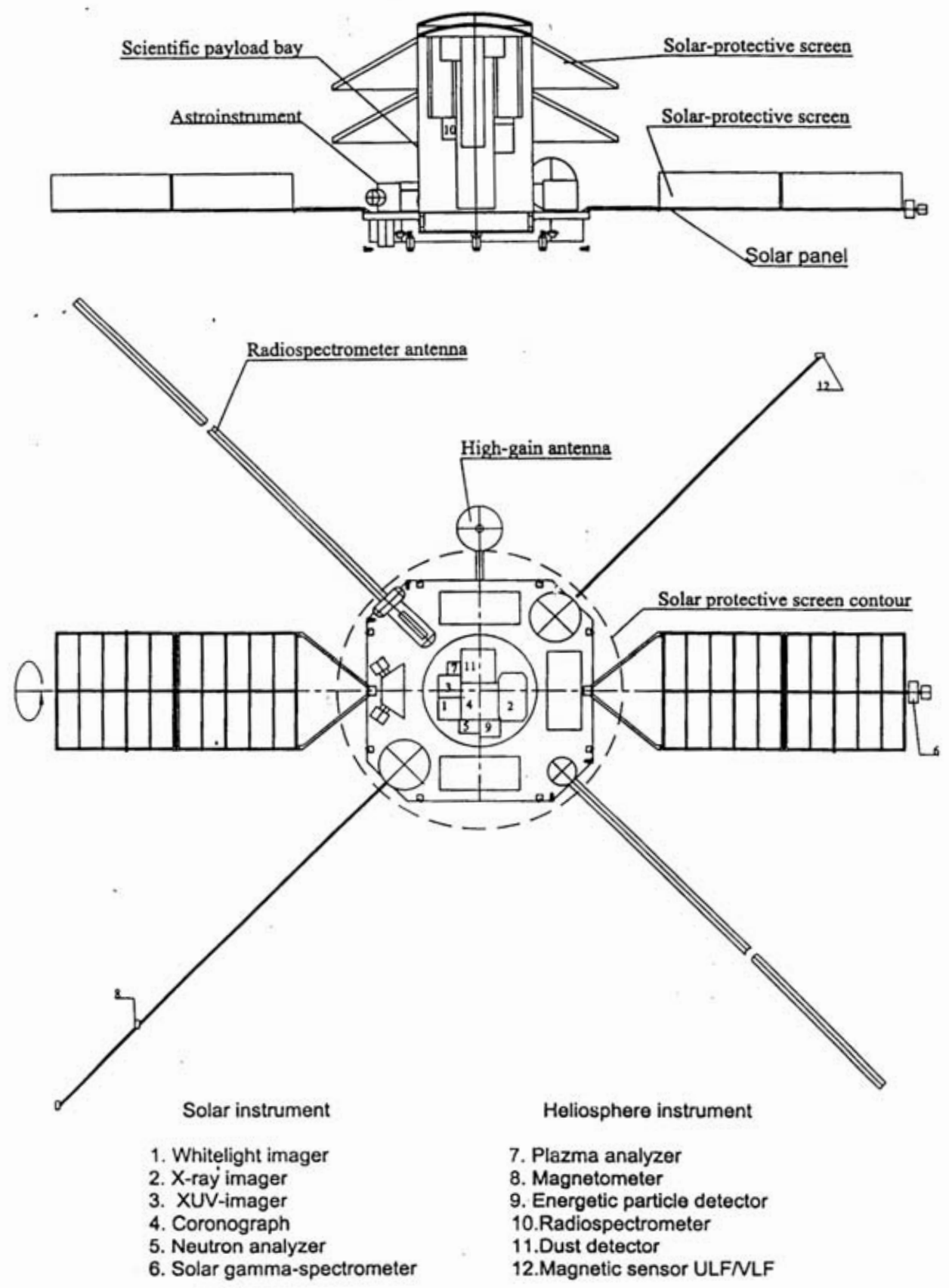

Figure 6. INTERHELIOPROBE spacecraft 


\section{THERION-F2 project}

The main objectives of the THERION-F2 (THERmosphere-IONosphere-F2) project are

- Monitoring of the environment on a basis of measurements of main parameters of neutral atmosphere and ionosphere of the Earth,

- Research of the physical nature of the ionosphere-thermosphere coupling in the planetary scale,

- Testing and development of the existing empirical and theoretical thermosphereionosphere models,

Prototype of THERION-F2 is the TIMED (Thermosphere Ionosphere Mesosphere Energetics and Dynamics) mission. An advantage of the project is a greater range of altitudes $(70-700 \mathrm{~km})$ studied by satellite at an elliptical orbit. The satellite periodically will measure the ionosphere parameters at a level of F2 layer: at the altitude $70-300 \mathrm{~km}$ by remote methods and at the altitude $350-700 \mathrm{~km}$ by contact (in situ) methods. The project will operate simultaneously with measurements of the non-coherent scattering experimental arrangement such as EISCAT.

Scientific payload (weight is $200 \mathrm{~kg}$ ) includes ionozond, neutral mass-spectrometer, Doppler interferometer for measuring of the neutral wind speed, accelerometer for measurement of the atmosphere density, electric field sensor, energetic particles sensors and photometer for measurement of the UV solar flux.

\section{Conclusions}

Projects discussed above have been prepared by scientific and space industry organizations and submitted to Space Research Council of Russian Academy of Sciences for expertise and recommendations for accomplishment in a framework of Russian Federal Space Program.

\section{Acknowledgements}

Paper is supported in part by Physical Department of Russian Academy of Sciences, Program N 18, INTAS, grant 03-51-3738 and RFBR, grants 04-02-16131-a and 04-0216152.

\section{References}

Demekhov, A.G., Trakhtengerts, V.Y., Mogilevsky, M.M., Zelenyi, L.M. 2003 Advances in Space Research 32, Issue 3, p. 355-374

Mogilevsky, M.M. 2002 , in Proc. of RF Ionospheric Interaction, 349-354, Santa Fe, New Mexico, 2002.

Oraevsky, V.N., Galeev, A.A., Kuznetsov, V.D., Zelenyi, L.M. 2001 Proceedings of Solar Encounter: The first solar orbiter workshop. Spain, 14-18 May 2001. ESA SP-493, p. 95-108

Oraevsky, V.N., Galeev, A.A., Kuznetsov, V.D., Zelenyi, L.M. 2002 Advances in Space Research 29, Issue 12, p. 2041-2050

Zelenyi, L.M., Petrukovich, A.A. 2004 Prospects of Russian Participation in International LWS Program Advances in Space Research (in press) 